\title{
Invariant mean and a Korovkin-type approximation theorem
}

\section{Saleh Abdullah Al-Mezel}

\section{"Correspondence:}

salmezel@kau.edu.sa

Department of Mathematics, King Abdulaziz University, P.O. Box 80203,

Jeddah, 21589, Saudi Arabia

\begin{abstract}
In this paper we apply this form of convergence to prove some Korovkin-type approximation theorem by using the test functions $1, e^{-x}, e^{-2 x}$, which generalizes the results of Boyanov and Veselinov (Bull. Math. Soc. Sci. Math. Roum. 14(62):9-13, 1970). MSC: $41 \mathrm{~A} 65 ; 46 \mathrm{A03} ; 47 \mathrm{H} 10 ; 54 \mathrm{H} 25$
\end{abstract}

Keywords: invariant mean; $\sigma$-convergence; Korovkin-type approximation theorem

\section{Introduction and preliminaries}

Let $c$ and $\ell_{\infty}$ denote the spaces of all convergent and bounded sequences, respectively, and note that $c \subset \ell_{\infty}$. In the theory of sequence spaces, an application of the well-known HahnBanach extension theorem gave rise to the concept of the Banach limit. That is, the lim functional defined on $c$ can be extended to the whole of $\ell_{\infty}$ and this extended functional is known as the Banach limit. In 1948, Lorentz [1] used this notion of a generalized limit to define a new type of convergence, known as almost convergence. Later on, Raimi [2] gave a slight generalization of almost convergence and named it $\sigma$-convergence. Before proceeding further, we recall some notations and basic definitions used in this paper.

Let $\sigma$ be a mapping of the set of positive integers into itself. A continuous linear functional $\varphi$ defined on the space $\ell_{\infty}$ of all bounded sequences is called an invariant mean (or a $\sigma$-mean; $c f$. [2]) if it is non-negative, normal and $\varphi(x)=\varphi\left(\left(x_{\sigma(n)}\right)\right)$.

A sequence $x=\left(x_{k}\right)$ is said to be $\sigma$-convergent to the number $L$ if and only if all of its $\sigma$ means coincide with $L$, i.e., $\varphi(x)=L$ for all $\varphi$. A bounded sequence $x=\left(x_{k}\right)$ is $\sigma$-convergent (cf. [3]) to the number $L$ if and only if $\lim _{p \rightarrow \infty} t_{p m}=L$ uniformly in $m$, where

$$
t_{p m}=\frac{x_{m}+x_{\sigma(m)}+x_{\sigma^{2}(m)}+\cdots+x_{\sigma p(m)}}{p+1} .
$$

We denote the set of all $\sigma$-convergent sequences by $V_{\sigma}$ and in this case we write $x_{k} \rightarrow$ $L\left(V_{\sigma}\right)$ and $L$ is called the $\sigma$-limit of $x$. Note that a $\sigma$-mean extends the limit functional on $c$ in the sense that $\varphi(x)=\lim x$ for all $x \in c$ if and only if $\sigma$ has no finite orbits (cf. [4]) and $c \subset V_{\sigma} \subset \ell_{\infty}$.

If $\sigma$ is a translation then the $\sigma$-mean is called a Banach limit and $\sigma$-convergence is reduced to the concept of almost convergence introduced by Lorentz [1].

In [5], the idea of statistical $\sigma$-convergence is defined which is further applied to prove some approximation theorems in [6] and [7].

o 2013 Al-Mezel; licensee Springer. This is an Open Access article distributed under the terms of the Creative Commons Attribution License (http://creativecommons.org/licenses/by/2.0), which permits unrestricted use, distribution, and reproduction in any medium, provided the original work is properly cited. 
If $m=1$, then we get $(C, 1)$ convergence, and in this case we write $x_{k} \rightarrow \ell(C, 1)$, where $\ell=(C, 1)-\lim x$.

Remark 1.1 Note that

(a) a convergent sequence is also $\sigma$-convergent;

(b) a $\sigma$-convergent sequence implies $(C, 1)$ convergence.

Example 1.1 Let $\sigma(n)=n+1$. Define the sequence $z=\left(z_{n}\right)$ by

$$
z_{n}= \begin{cases}1 & \text { if } n \text { is odd } \\ 0 & \text { if } n \text { is even }\end{cases}
$$

Then $x$ is $\sigma$-convergent to $1 / 2$ but not convergent.

Let $C[a, b]$ be the space of all functions $f$ continuous on $[a, b]$. We know that $C[a, b]$ is a Banach space with the norm $\|f\|_{\infty}:=\sup _{a \leq x \leq b}|f(x)|, f \in C[a, b]$. Suppose that $T_{n}$ : $C[a, b] \rightarrow C[a, b]$. We write $T_{n}(f, x)$ for $T_{n}(f(t), x)$ and we say that $T$ is a positive operator if $T(f, x) \geq 0$ for all $f(x) \geq 0$.

The classical Korovkin approximation theorem states the following [7]: Let $\left(T_{n}\right)$ be a sequence of positive linear operators from $C[a, b]$ into $C[a, b]$. Then $\lim _{n}\left\|T_{n}(f, x)-f(x)\right\|_{\infty}=$ 0 , for all $f \in C[a, b]$ if and only if $\lim _{n}\left\|T_{n}\left(f_{i}, x\right)-f_{i}(x)\right\|_{\infty}=0$, for $i=0,1,2$, where $f_{0}(x)=1$, $f_{1}(x)=x$ and $f_{2}(x)=x^{2}$.

Quite recently, such type of approximation theorem has been studied in [8,9] and [10] by using $\lambda$-statistical convergence, while in [11] lacunary statistical convergence has been used. Boyanov and Veselinov [12] have proved the Korovkin theorem on $C[0, \infty)$ by using the test functions $1, e^{-x}, e^{-2 x}$. In this paper, we generalize the result of Boyanov and Veselinov by using the notion of $\sigma$-convergence. Our results also generalize the results of Mohiuddine [13], in which the author has used almost convergence and the test functions $1, x, x^{2}$.

\section{Korovkin-type approximation theorem}

We prove the following $\sigma$-version of the classical Korovkin approximation theorem.

Theorem 2.1 Let $\left(T_{k}\right)$ be a sequence of positive linear operators from $C(I)$ into $C(I)$. Then, for all $f \in C(I)$,

$$
\sigma-\lim _{k \rightarrow \infty}\left\|T_{k}(f ; x)-f(x)\right\|_{\infty}=0
$$

if and only if

$$
\begin{aligned}
& \sigma-\lim _{k \rightarrow \infty}\left\|T_{k}(1 ; x)-1\right\|_{\infty}=0, \\
& \sigma-\lim _{k \rightarrow \infty}\left\|T_{k}\left(e^{-s} ; x\right)-e^{-x}\right\|_{\infty}=0, \\
& \sigma-\lim _{k \rightarrow \infty}\left\|T_{k}\left(e^{-2 s} ; x\right)-e^{-2 x}\right\|_{\infty}=0 .
\end{aligned}
$$


Proof Since each $1, e^{-x}, e^{-2 x}$ belongs to $C(I)$, conditions (2.2)-(2.4) follow immediately from (2.1). Let $f \in C(I)$. Then there exists a constant $M>0$ such that $|f(x)| \leq M$ for $x \in I$. Therefore,

$$
|f(s)-f(x)| \leq 2 M, \quad-\infty<s, x<\infty \text {. }
$$

It is easy to prove that for a given $\varepsilon>0$ there is a $\delta>0$ such that

$$
|f(s)-f(x)|<\varepsilon,
$$

whenever $\left|e^{-s}-e^{-x}\right|<\delta$ for all $x \in I$.

Using (2.5), (2.6), putting $\psi_{1}=\psi_{1}(s, x)=\left(e^{-s}-e^{-x}\right)^{2}$, we get

$$
|f(s)-f(x)|<\varepsilon+\frac{2 M}{\delta^{2}}\left(\psi_{1}\right), \quad \forall|s-x|<\delta .
$$

This is,

$$
-\varepsilon-\frac{2 M}{\delta^{2}}\left(\psi_{1}\right)<f(s)-f(x)<\varepsilon+\frac{2 M}{\delta^{2}}\left(\psi_{1}\right) .
$$

Now, we operate $T_{\sigma^{k}(n)}(1, x)$ for all $n$ to this inequality since $T_{\sigma^{k}(n)}(f, x)$ is monotone and linear. We obtain

$$
\begin{aligned}
T_{\sigma^{k}(n)}(1 ; x)\left(-\varepsilon-\frac{2 M}{\delta^{2}}\left(\psi_{1}\right)\right) & <T_{\sigma^{k}(n)}(1 ; x)(f(s)-f(x)) \\
& <T_{\sigma^{k}(n)}(1 ; x)\left(\varepsilon+\frac{2 M}{\delta^{2}}\left(\psi_{1}\right)\right) .
\end{aligned}
$$

Note that $x$ is fixed and so $f(x)$ is a constant number. Therefore

$$
\begin{aligned}
-\varepsilon T_{\sigma^{k}(n)}(1 ; x)-\frac{2 M}{\delta^{2}} T_{\sigma^{k}(n)}\left(\psi_{1} ; x\right) & <T_{\sigma^{k}(n)}(f ; x)-f(x) T_{\sigma^{k}(n)}(1 ; x) \\
& <\varepsilon T_{\sigma^{k}(n)}(1 ; x)+\frac{2 M}{\delta^{2}} T_{\sigma^{k}(n)}\left(\psi_{1} ; x\right) .
\end{aligned}
$$

But

$$
\begin{aligned}
& T_{\sigma^{k}(n)}(f ; x)-f(x) \\
& \quad=T_{\sigma^{k}(n)}(f ; x)-f(x) T_{\sigma^{k}(n)}(1 ; x)+f(x) T_{\sigma^{k}(n)}(1 ; x)-f(x) \\
& \quad=\left[T_{\sigma^{k}(n)}(f ; x)-f(x) T_{\sigma^{k}(n)}(1 ; x)\right]+f(x)\left[T_{\sigma^{k}(n)}(1 ; x)-1\right] .
\end{aligned}
$$

Using (2.7) and (2.8), we have

$$
\begin{aligned}
T_{\sigma^{k}(n)}(f ; x)-f(x)< & \varepsilon T_{\sigma^{k}(n)}(1 ; x)+\frac{2 M}{\delta^{2}} T_{\sigma^{k}(n)}\left(\psi_{1} ; x\right) \\
& +f(x)\left(T_{\sigma^{k}(n)}(1 ; x)-1\right) .
\end{aligned}
$$


Now

$$
\begin{aligned}
T_{\sigma^{k}(n)}\left(\psi_{1} ; x\right)= & T_{\sigma^{k}(n)}\left(\left(e^{-s}-e^{-x}\right)^{2} ; x\right)=T_{\sigma^{k}(n)}\left(e^{-2 s}-2 e^{-s} e^{-x}+e^{-2 x} ; x\right) \\
= & T_{\sigma^{k}(n)}\left(e^{-2 s} ; x\right)-2 e^{-x} T_{\sigma^{k}(n)}\left(e^{-s} ; x\right)+\left(e^{-2 x}\right) T_{\sigma^{k}(n)}(1 ; x) \\
= & {\left[T_{\sigma^{k}(n)}\left(e^{-2 s} ; x\right)-e^{-2 x}\right]-2 e^{-x}\left[T_{\sigma^{k}(n)}\left(e^{-s} ; x\right)-e^{-x}\right] } \\
& +e^{-2 x}\left[T_{\sigma^{k}(n)}(1 ; x)-1\right] .
\end{aligned}
$$

Using (2.9), we obtain

$$
\begin{aligned}
T_{\sigma^{k}(n)}(f ; x)-f(x)< & \varepsilon T_{\sigma^{k}(n)}(1 ; x)+\frac{2 M}{\delta^{2}}\left\{\left[T_{\sigma^{k}(n)}\left(\left(e^{-2 s}\right) ; x\right)-e^{-2 x}\right]\right. \\
& \left.-2 e^{-x}\left[T_{\sigma^{k}(n)}\left(e^{-s} ; x\right)-e^{-x}\right]+e^{-2 x}\left[T_{\sigma^{k}(n)}(1 ; x)-1\right]\right\} \\
& +f(x)\left(T_{\sigma^{k}(n)}(1 ; x)-1\right) \\
= & \varepsilon\left[T_{\sigma^{k}(n)}(1 ; x)-1\right]+\varepsilon+\frac{2 M}{\delta^{2}}\left\{\left[T_{\sigma^{k}(n)}\left(\left(e^{-2 s}\right) ; x\right)-e^{-2 x}\right]\right. \\
& \left.-2 e^{-x}\left[T_{\sigma^{k}(n)}\left(e^{-s} ; x\right)-e^{-x}\right]+e^{-2 x}\left[T_{\sigma^{k}(n)}(1 ; x)-1\right]\right\} \\
& +f(x)\left(T_{\sigma^{k}(n)}(1 ; x)-1\right) .
\end{aligned}
$$

Since $\varepsilon$ is arbitrary, we can write

$$
\begin{aligned}
T_{\sigma^{k}(n)}(f ; x)-f(x) \leq & \varepsilon\left[T_{\sigma^{k}(n)}(1 ; x)-1\right]+\frac{2 M}{\delta^{2}}\left\{\left[T_{\sigma^{k}(n)}\left(\left(e^{-2 s}\right) ; x\right)-e^{-2 x}\right]\right. \\
& \left.-2 e^{-x}\left[T_{\sigma^{k}(n)}\left(e^{-s} ; x\right)-e^{-x}\right]+e^{-2 x}\left[T_{\sigma^{k}(n)}(1 ; x)-1\right]\right\} \\
+ & f(x)\left[T_{\sigma^{k}(n)}(1 ; x)-1\right] .
\end{aligned}
$$

Therefore

$$
\begin{aligned}
\left|T_{\sigma^{k}(n)}(f ; x)-f(x)\right| & \\
\leq & \varepsilon+(\varepsilon+M)\left|T_{\sigma^{k}(n)}(1 ; x)-1\right|+\frac{2 M}{\delta^{2}}\left|e^{-2 x}\right|\left|T_{\sigma^{k}(n)}(1 ; x, y)-1\right| \\
& +\frac{2 M}{\delta^{2}}\left|T_{\sigma^{k}(n)}\left(e^{-2 s} ; x\right)\right|\left|-e^{-2 x}\right|+\frac{4 M}{\delta^{2}}\left|e^{-x}\right|\left|T_{\sigma^{k}(n)}\left(e^{-s} ; x\right)-e^{-x}\right| \\
\leq & +\left(\varepsilon+M+\frac{4 M}{\delta^{2}}\right)\left|T_{\sigma^{k}(n)}(1 ; x)-1\right|+\frac{2 M}{\delta^{2}}\left|e^{-2 x}\right|\left|T_{\sigma^{k}(n)}(1 ; x)-1\right| \\
& +\frac{2 M}{\delta^{2}}\left|T_{\sigma^{k}(n)}\left(e^{-2 s} ; x\right)-e^{-2 x}\right|+\frac{4 M}{\delta^{2}}\left|T_{\sigma^{k}(n)}\left(e^{-s} ; x\right)-e^{-x}\right|
\end{aligned}
$$

since $\left|e^{-x}\right| \leq 1$ for all $x \in I$. Now, taking $\sup _{x \in I}$

$$
\begin{aligned}
\left\|T_{\sigma^{k}(n)}(f ; x)-f(x)\right\|_{\infty} \leq & \varepsilon+K\left(\left\|T_{\sigma^{k}(n)}(1 ; x)-1\right\|_{\infty}+\left\|T_{\sigma^{k}(n)}\left(e^{-s} ; x\right)-e^{-x}\right\|_{\infty}\right. \\
& \left.+\left\|T_{\sigma^{k}(n)}\left(e^{-2 s} ; x\right)-e^{-2 x}\right\|_{\infty}\right),
\end{aligned}
$$


where $K=\max \left\{\varepsilon+M+\frac{4 M}{\delta^{2}}, \frac{2 M}{\delta^{2}}\right\}$. Now writing

$$
D_{n, p}(f, x)=\frac{1}{p} \sum_{k=0}^{p-1} T_{\sigma^{k}(n)}(f, x)
$$

we get

$$
\begin{aligned}
\left\|D_{n, p}(f, x)-f(x)\right\|_{\infty} \leq & \left(\epsilon+\frac{2 M b^{2}}{\delta^{2}}+M\right)\left\|D_{n, p}(1, x)-1\right\|_{\infty} \\
& +\frac{4 M b}{\delta^{2}}\left\|D_{n, p}(t, x)-e^{-x}\right\|_{\infty}+\frac{2 M}{\delta^{2}}\left\|D_{n, p}\left(t^{2}, x\right)-e^{-2 x}\right\|_{\infty} .
\end{aligned}
$$

Letting $p \rightarrow \infty$ and using (2.2), (2.3), (2.4), we get

$$
\lim _{p \rightarrow \infty}\left\|D_{n, p}(f, x)-f(x)\right\|_{\infty}=0, \quad \text { uniformly in } n
$$

In the following example we construct a sequence of positive linear operators satisfying the conditions of Theorem 2.1 but not satisfying the conditions of the Korovkin theorem of Boyanov and Veselinov [12].

Example 2.1 Consider the sequence of classical Baskakov operators [14]

$$
V_{n}(f ; x):=\sum_{k=0}^{\infty} f\left(\frac{k}{n}\right)\left(\begin{array}{c}
n-1+k \\
k
\end{array}\right) x^{k}(1+x)^{-n-k}
$$

where $0 \leq x, y<\infty$.

Let the sequence $\left(L_{n}\right)$ be defined by $L_{n}: C(I) \rightarrow C(I)$ with $L_{n}(f ; x)=\left(1+z_{n}\right) V_{n}(f ; x)$, where $z_{n}$ is defined as above. Since

$$
\begin{aligned}
& L_{n}(1 ; x)=1, \\
& L_{n}\left(e^{-s} ; x\right)=\left(1+x-x e^{-\frac{1}{n}}\right)^{-n}, \\
& L_{n}\left(e^{-2 s} ; x\right)=\left(1+x^{2}-x^{2} e^{-\frac{1}{n}}\right)^{-n},
\end{aligned}
$$

and the sequence $\left(P_{n}\right)$ satisfies the conditions (2.1), (2.2) and (2.3). Hence we have

$$
\sigma-\lim \left\|L_{n}(f, x)-f(x)\right\|_{\infty}=0
$$

On the other hand, we get $L_{n}(f, 0)=\left(1+z_{n}\right) f(0)$ since $L_{n}(f, 0)=f(0)$, and hence

$$
\left\|L_{n}(f, x)-f(x)\right\|_{\infty} \geq\left|L_{n}(f, 0)-f(0)\right|=z_{n}|f(0)| .
$$

We see that $\left(L_{n}\right)$ does not satisfy the classical Korovkin theorem since lim $\sup _{n \rightarrow \infty} z_{n}$ does not exist. Hence our Theorem 2.1 is stronger than that of Boyanov and Veselinov $[12]$. 


\section{A consequence}

Now we present a slight general result.

Theorem 3.1 Let $\left(T_{n}\right)$ be a sequence of positive linear operators on $C(I)$ such that

$$
\limsup _{n} \frac{1}{n} \sum_{k=0}^{n-1}\left\|T_{n}-T_{\sigma^{k}(m)}\right\|=0
$$

If

$$
\sigma-\lim _{n}\left\|T_{n}\left(e^{-v s}, x\right)-e^{-v x}\right\|_{\infty}=0 \quad(v=0,1,2),
$$

then, for any function $f \in C(I)$ bounded on the real line, we have

$$
\lim _{n}\left\|T_{n}(f, x)-f(x)\right\|_{\infty}=0
$$

Proof From Theorem 2.1, we have that if (3.1) holds, then

$$
\sigma-\lim _{n}\left\|T_{n}(f, x)-f(x)\right\|_{\infty}=0
$$

which is equivalent to

$$
\lim _{n}\left\|\sup _{m} D_{m, n}(f, x)-f(x)\right\|_{\infty}=0
$$

Now

$$
\begin{aligned}
T_{n}-D_{m, n} & =T_{n}-\frac{1}{n} \sum_{k=0}^{n-1} T_{\sigma^{k}(m)} \\
& =\frac{1}{n} \sum_{k=0}^{n-1}\left(T_{n}-T_{\sigma^{k}(m)}\right) .
\end{aligned}
$$

Therefore

$$
T_{n}-\sup _{m} D_{m, n}=\sup _{m} \frac{1}{n} \sum_{k=0}^{n-1}\left(T_{n}-T_{\sigma^{k}(m)}\right) .
$$

Hence, using the hypothesis, we get

$$
\lim _{n}\left\|T_{n}(f, x)-f(x)\right\|_{\infty}=\lim _{n}\left\|\sup _{m} D_{m, n}(f, x)-f(x)\right\|_{\infty}=0,
$$

that is, (3.2) holds.

\section{Competing interests}

The author declares that they have no competing interests. 


\section{References}

1. Lorentz, GG: A contribution to theory of divergent sequences. Acta Math. 80, 167-190 (1948)

2. Raimi, RA: Invariant means and invariant matrix methods of summability. Duke Math. J. 30, 81-94 (1963)

3. Schaefer, P: Infinite matrices and invariant means. Proc. Am. Math. Soc. 36, 104-110 (1972)

4. Mursaleen, M: On some new invariant matrix methods of summability. Quart. J. Math. Oxford 34, 77-86 (1983)

5. Mursaleen, M, Edely, OHH: On the invariant mean and statistical convergence. Appl. Math. Lett. 22, $1700-1704$ (2009)

6. Demirci, K, Dirik, F: Statistical $\sigma$-convergence of positive linear operators. Appl. Math. Lett. 24, 375-380 (2011)

7. Karakuş, S, Demirci, K: Equi-statistical $\sigma$-convergence of positive linear operators. Comput. Math. Appl. 60, 2212-2218 (2010)

8. Edely, $\mathrm{OHH}$, Mohiuddine, SA, Noman, AK: Korovkin type approximation theorems obtained through generalized statistical convergence. Appl. Math. Lett. 23, 1382-1387 (2010)

9. Mursaleen, M, Alotaibi, A: Statistical summability and approximation by de la Vallée-Pousin mean. Appl. Math. Lett. 24, 320-324 (2011)

10. Srivastava, HM, Mursaleen, M, Khan, A: Generalized equi-statistical convergence of positive linear operators and associated approximation theorems. Math. Comput. Model. 55, 2040-2051 (2012)

11. Mursaleen, M, Alotaibi, A: Statistical lacunary summability and a Korovkin type approximation theorem. Ann. Univ Ferrara 57(2), 373-381 (2011)

12. Boyanov, BD, Veselinov, VM: A note on the approximation of functions in an infinite interval by linear positive operators. Bull. Math. Soc. Sci. Math. Roum. 14(62), 9-13 (1970)

13. Mohiuddine, SA: An application of almost convergence in approximation theorems. Appl. Math. Lett. 24, 1856-1860 (2011)

14. Becker, M: Global approximation theorems for Szasz-Mirakjan and Baskakov operators in polynomial weight spaces. Indiana Univ. Math. J. 27(1), 127-142 (1978)

doi:10.1186/1029-242X-2013-103

Cite this article as: Al-Mezel: Invariant mean and a Korovkin-type approximation theorem. Journal of Inequalities and Applications 2013 2013:103.

\section{Submit your manuscript to a SpringerOpen ${ }^{0}$ journal and benefit from:}

- Convenient online submission

- Rigorous peer review

- Immediate publication on acceptance

- Open access: articles freely available online

- High visibility within the field

- Retaining the copyright to your article 